\title{
Next generation sequencing as second-tier test in high-throughput newborn screening for nephropathic cystinosis
}

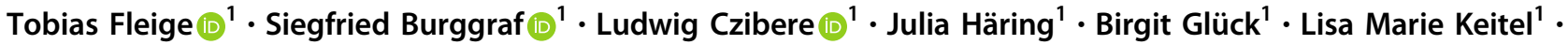 \\ Olfert Landt $^{2}$ - Erik Harms ${ }^{3} \cdot$ Katharina Hohenfellner $^{4} \cdot$ Jürgen Durner ${ }^{1,5} \cdot$ Wulf Röschinger $^{1} \cdot$ Marc Becker $^{1,5}$
}

Received: 2 April 2019 / Revised: 11 September 2019 / Accepted: 17 September 2019 / Published online: 30 September 2019

(c) The Author(s), under exclusive licence to European Society of Human Genetics 2019

\begin{abstract}
Nephropathic cystinosis is a rare autosomal recessive lysosomal storage disorder, which causes loss of renal proximal tubular function and progressive loss of glomerular function, finally leading to end stage renal failure at school age. In the course of the disease most patients will need kidney transplantation if treatment has not been started before clinical manifestation. With an effective treatment available, a newborn screening assay is highly demanded. Since newborns with cystinosis usually do not show symptoms within the first months of life and no biochemical markers are easily detectable, a DNA-based method seems to be an obvious tool for early diagnosis. Screening was performed using high-throughput nucleic acid extraction followed by 384-well qPCR and melting analysis for the three most frequent variants $(57 \mathrm{~kb}$ deletion NC_000017.11:g.3600934_3658165del (GRCh38); c.18_21del GACT; c.926dupG) responsible for the defective lysosomal membrane protein cystinosin (CTNS). To increase sensitivity, all heterozygous samples identified in qPCR assay were verified and screened for additional variants by applying next generation sequencing. From January 2018 to July 2019 nearly 292,000 newborns were successfully screened. We identified two newborns with a homozygous $57 \mathrm{~kb}$ deletion and a second one with heterozygous $57 \mathrm{~kb}$ deletion and a $\mathrm{G}>\mathrm{C}$ substitution at position c.-512 on the second allele. Cystinosis is an example for diseases caused by a limited number of high prevalence and a high number of low prevalence variants. We have shown that qPCR combined with NGS can be used as a high throughput, cost effective tool in newborn screening for such diseases.
\end{abstract}

Supplementary information The online version of this article (https:// doi.org/10.1038/s41431-019-0521-3) contains supplementary material, which is available to authorized users.

Tobias Fleige

t.fleige@labor-becker.de

1 Laboratory Becker \& Colleagues, Führichstr. 70, 81671 Munich, Germany

2 TIB Molbiol GmbH, Eresburgstr. 22-23, 12103 Berlin, Germany

3 University Hospital Münster, Albert-Schweitzer-Campus 1, 48149 Münster, Germany

4 Pediatric Nephrology, RoMed Klinikum Rosenheim, Pettenkoferstr. 10, 83022 Rosenheim, Germany

5 Department of Operative/Restorative Dentistry, Periodontology and Pedodontics, Ludwig Maximilian University Munich, Goethestr. 70, 80337 Munich, Germany

\section{Introduction}

Newborn screening (NBS) is a successful public health program preventing morbidity and mortality through early diagnosis and management of conditions including rare inborn errors of metabolism [1]. Mass screening of newborns with dried blood spots (DBS) started in the early 1960s based on a method established by Guthrie and Susi to easily identify patients with phenylketonuria [2]. With the introduction of tandem mass spectrometry (MS/MS) up to 49 metabolic inborn errors can be identified from a single blot spot today [3]. The German newborn screening program includes 14 main category disorders.

Nephropathic cystinosis is a rare autosomal recessive lysosomal storage disorder, with an estimated frequency of 1:100,000-1:200,000. It is caused by missing or nonfunctional cystinosin protein (CTNS, OMIM: 606272) leading to the accumulation of free cystine in lysosomes of various tissue types [4]. The major clinical symptoms are renal failure, growth retardation, and rickets. The therapeutic 
approach is to deplete the lysosomal cystine by administering aminothiol cysteamine, the only available drug to date. Used as a target specific treatment, it leads to lysosomal depletion in all types of cells and tissues in the body by a disulfide exchange reaction with cystine. If treatment is started as soon as possible, best directly after birth [4, 5], progression to end stage renal disease is dramatically reduced or even prevented [6]. Although accumulation of cystine already starts in utero, first symptoms usually do not appear before the age of six months [6]. Due to the low incidence of cystinosis, there is also the risk of it not being recognized immediately.

Since, to our knowledge, no method for standard newborn screening to identify cystinosis is available today, we decided to develop the first DNA-based approach for early diagnosis.

The CTNS gene is located on a $23 \mathrm{Mb}$ section of the short arm of chromosome 17 consisting of two noncoding and ten coding exons. In Northern Europe, the most common pathologic variation is a $57 \mathrm{~kb}$ (originally denominated as $65 \mathrm{~kb}$ ) deletion, OMIM \# 606272.0005, removing the promoter, exons 1-9, most of exon 10 [7], the sedoheptulokinase $(S H P K)$ gene, as well as leading to TRPVI dysfunction [8]. The deletion was putatively formed by nonhomologous recombination [9] with characteristic deletion breakpointsmost likely-identical for all patients and heterozygous carriers. Haplotype analysis showed that this deletion originates in Northern Europe sometime in the middle of the first millennium [10], leading to a high prevalence especially in Germany, reaching up to $65 \%$. More than 140 disease causing variants-homo or compound heterozygous -have been reported in the human gene mutation database $\left(H G M D^{\circledR}\right)$, mostly randomly distributed all through the genomic locus. Some variants affecting the function of cystinosin are overrepresented in specific geographic regions like c.18_21del GACT and c.926dupG in Germany and Switzerland or c.681 G>A in Turkey [11, 12]. So far, the use of DNA-based methods in NBS was mainly restricted to confirmatory assays in second or third-tier approaches, e.g., screening for Krabbe disease in New York State, or cystic fibrosis screening in Germany [13, 14]. In recent years, several countries established a screening for T-cell receptor excision circles (TREC) detection to identify newborns with defective maturation of cell-mediated immunity (severe combined immunodeficiency (SCID)). Although TREC screening is DNA-based, it does not include any personal sequence data. Therefore, it cannot be regarded as classic genetic screening. Some pilot studies used qPCR assays for the detection of a homozygous deletion of the SMN1 gene to diagnose spinal muscular atrophy (SMA; OMIM \#: 253300) and deafness-related variants of three genes responsible for congenital nonsyndromic hearing loss $[15,16]$. A common aspect of genetic NBS programs today is the focus on limited numbers of genetic modifications. For more complex genetic disorders, alternative methods like next-generation sequencing (NGS) have to be established.

NGS is a powerful diagnostic tool already used in several clinical settings e.g., in pathology for tumor characterization influencing therapeutic decisions or when using whole genome sequencing to help with the diagnosis of unknown conditions in newborns $[17,18]$. But it is still controversially discussed in the field of NBS, regarding ethical concerns, costs, legal issues, and reporting $[1,3]$.

Here we present a novel NBS algorithm, which combines qPCR including melting analysis with NGS. A highthroughput screening for the three most common variants of CTNS found in the German population followed by sequencing of heterozygous samples was implemented and tested with $>290,000$ samples. Including NGS as a second tier test to detect compound heterozygous samples, we increased sensitivity without significantly rising turnaround time or screening costs. We identified two newborns with a homozygous $57 \mathrm{~kb}$ deletion by qPCR only and a second one with heterozygous $57 \mathrm{~kb}$ deletion and a $\mathrm{G}>\mathrm{C}$ substitution at position c.-512 on the second allele, using qPCR and NGS.

\section{Material and methods}

\section{Sampling}

Our study started in January of 2018, encompassing samples from children mostly born in Bavaria and partly from other regions of Germany. According to German law, parents were informed and had to decide if they wanted to participate in the study (informed consent). The regional ethical board approved the study (Ethical permit 16125; Bayerische Landesärztekammer Munich). Blood samples were taken from the same DBS cards collected for classical NBS. No additional sampling was needed. $3.2 \mathrm{~mm}$ punches were collected in 96-well PCR plates (4ti-0770/C, 4titude, Surrey, UK) using an automated Panthera Puncher 9 System (PerkinElmer, Waltham, MA, USA). Alternating distribution of negative controls within the 96-well plates assured the unambiguous assignment of the plates.

\section{Nucleic acid isolation from DBS cards}

Briefly, DNA was extracted from single $3.2 \mathrm{~mm}$ punches of the original DBS in a 96-well format. After an incubation step with water, washing buffer was added. Following 10 min incubation, buffer was removed and the washing step repeated two times, first with washing buffer and finally with water. Elution was done with $50 \mu \mathrm{l} \mathrm{CE-Elution} \mathrm{buffer.}$ A detailed description of the procedure is published 
elsewhere [19]. For sequencing, a second DNA extraction from two punches was performed following the same procedure in order to ensure a higher quantity of DNA.

\section{MagNA Pure 96 nucleic acid isolation}

Nucleic acid from EDTA-blood was isolated using the MagNA Pure 96 system from Roche (Mannheim, Germany). A total of $200 \mu \mathrm{l}$ sample material was transferred in 96-deepwell plate (Roche) by a primary sampling handling unit (Roche). For nucleic acid isolation, the DNA/ Viral NA Small Volume 2.0 Kit following the Pathogen Universal 2004.0 protocol with $50 \mu \mathrm{l}$ elution volume was used.

\section{qPCR screening assay}

The $57 \mathrm{~kb}$ deletion (NC_000017.11:g.3600934_3658165del (GRCh38)) is detected using forward primer 5'- CCggCATAACCCACAATg located 327 bp downstream of the published primer LD1 [20] and reverse primer 5'CCACCggCCATgTgAA moved 25 bp upstream from the published primer, reducing the amplicon length from originally 1255 bp [9] or 423 bp [20] to $61 \mathrm{bp}$ only. Detection of the product is done with a LightCycler Red 610 labeled hydrolysis probe (ROX channel) (Fig S1). The second assay targets the c.18_21del GACT deletion located in exon 3 (rs786204501; reference sequence NM_001031681.2), combining a hydrolysis probe with LightCycler Red 640 label with a deletion-specific FRET donor probe with the sequence $5{ }^{\prime}$-gATAAggAATTgg__CTATTTTTATCC T-PH. The c.18_21del GACT variant is detected by running a melting analysis, reporting a high melting temperature $(\mathrm{Tm})$ in case of the deletion and a lower Tm peak for the wild type, or two peaks in case of a heterozygous deletion (Fig S1).

The exon 11 guanosine insertion (rs786204420; reference sequence NM_001031681.2) is detected from a 84 bp long PCR fragment using a SimpleProbe oligomer specific for the c.926dupG sequence TCACC_g_gggggCAgCTTCAgCCTC--PH, thus reporting the higher melting point for the variant, a lower Tm peak for the wild type, and two peaks in case of a heterozygous genotype (Fig S1).

\section{qPCR}

qPCR was performed on a 384-well LightCycler480II (Roche) using Multiplex DNA Master (Roche) in $10 \mu \mathrm{l}$ reactions and the following PCR profile: $95^{\circ} \mathrm{C} 10 \mathrm{~min}$ denaturation; $45 \times\left(95^{\circ} \mathrm{C} 5 \mathrm{~s} ; 60^{\circ} \mathrm{C} 10 \mathrm{~s}\right.$ [fluorescence measurement]; $\left.72{ }^{\circ} \mathrm{C} 15 \mathrm{~s}\right)$; Melting $\left(95^{\circ} \mathrm{C} 30 \mathrm{~s} ; 40^{\circ} \mathrm{C}\right.$ $2 \mathrm{~min} ; 85^{\circ} \mathrm{C}$ continuous fluorescent measurement with a ramping rate of $0.11^{\circ} \mathrm{C} / \mathrm{s}$ ). Sample lists were generated from punching $\log$ files and imported into the
LightCycler480II. For all samples showing a variant or inconclusive result, the assay was repeated with two fresh punches from the original DBS card.

\section{NGS assay}

NGS was done on an Illumina MiniSeq (Illumina GmbH, Munich, Germany) using a midoutput cartridge and $2 \times 151$ bp read length. The TrueSeq Custom Amplicon Low Input Assay covered the complete coding region of the CTNS gene, including exon-intron boundaries as well as the $5^{\prime}$ and $3^{\prime}$ untranslated region. Library preparation was done according to manufacturer's instructions. In total, 29 amplicons were sequenced per sample. Sequence analysis was performed with the Integrative Genomics Viewer (IGV) 2.4 [21, 22] and Illumina Variant Studio 3.0 (Illumina $\mathrm{GmbH}$ ). Sequences were submitted to SRA database (https://www.ncbi.nlm.nih. gov/sra/ accession number: PRJNA564496).

\section{Results}

\section{qPCR validation}

Presence of the $57 \mathrm{~kb}$ deletion is detected through an amplification signal giving a crossing point around cq 27. As the primer binding sites are close to the breakpoints of the deletion a PCR product is only produced when the $57 \mathrm{~kb}$ deletion is present (Fig. 1a). For differentiation between homozygous or heterozygous state, a second channel is needed. Therefore the region around the c.18_21del GACT is amplified, located within the $57 \mathrm{~kb}$ deletion of the CTNS gene. Amplification of this part allows not only the differentiation between heterozygous and homozygous state of $57 \mathrm{~kb}$ deletion due to the presence or absence of amplification (Fig. 1b). It also serves as isolation and amplification control and therefore controlling the PCR reaction. Amplification failure in the differentiation channel without a positive signal for the $57 \mathrm{~kb}$ deletion would invalidate the assay.

The presence of the c.18_21del GACT (rs786204501) deletion is detected via melting point analysis. Wildtypedeletion negative - samples show a melting peak around $46{ }^{\circ} \mathrm{C}$, whereas in presence of the deletion a higher melting peak is visible at $55^{\circ} \mathrm{C}$. Differentiation between homo- and heterozygous states is demonstrated by one or two melting peaks (Fig. 1c). Applying the same method, the insertion of a single guanosine at position c.926 (rs786204420) is detected, leading again to a shift in the melting peak from 65 to $72{ }^{\circ} \mathrm{C}$ (Fig. 1d). All three variants are detected within one single multiplex qPCR, still leaving two channels open for other assays.

Twenty blood samples provided from patients suffering from cystinosis were used for assay validation. DNA was 

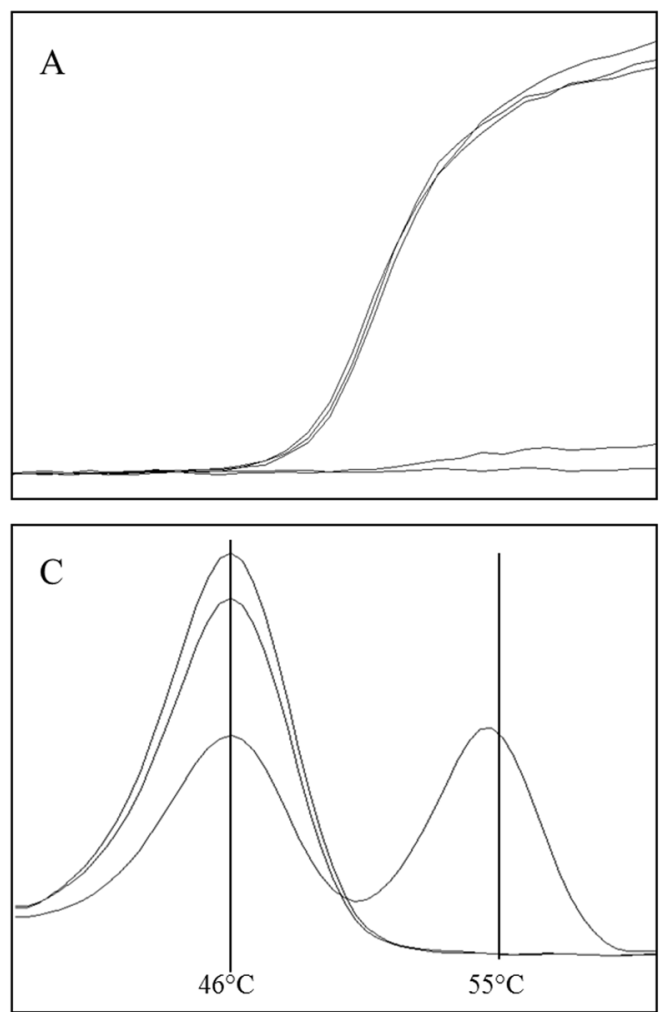

Fig. 1 Multiplex PCR for simultaneously detection of CTNS variants. a Amplification signal for $57 \mathrm{~kb}$ deletion, detection filter 533-610, three samples show an exponential amplification signal, demonstrating the presence of the deletion. One sample was negative in this assay and the last one represents the negative control, both without amplification signal. No differentiation between homo- or heterozygous state is possible. b Amplification of parts of exon 3 used for differentiation between a homo- or heterozygous $57 \mathrm{~kb}$ deletion as well as amplification control (Channel 533-610). One sample gives an exponential amplification signal, indicating the presence of the wildtype region used for differentiation, two samples were negative and one is the

isolated using the MagNA Pure 96 system. All samples had previously been characterized by an independent laboratory using MLPA or sequence analysis. Four samples showed a clear amplification signal for the $57 \mathrm{~kb}$ deletion and an allelic drop-out in the differentiation channel, pointing to a homozygous deletion. Four other samples were homozygous for the c.18_21del GACT deletion and one homozygous for c.926dupG. From the remaining eleven samples, ten were heterozygous for the $57 \mathrm{~kb}$ deletion-one compound heterozygous for the c.18_21del GACT and one for the c.926dupG. One sample had none of the three variants screened for. All qPCR results were in concordance with the previous analysis (Tab. 1)

\section{qPCR robustness}

The amount of samples to be repeated is an indication of the qPCR robustness. The rate of invalid results due to $\mathrm{qPCR}$
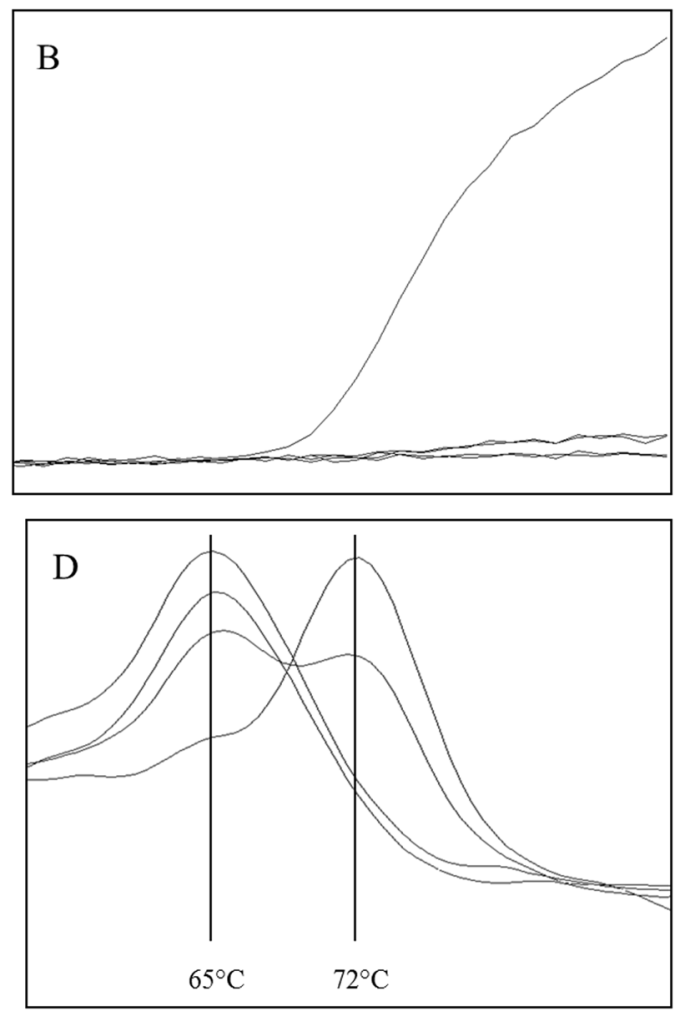

negative control. c Detection of rs786204501 (c.18_21del GACT) via melting analysis (Channel 498-640). The mutated allele gives a melting peak of app. $55^{\circ} \mathrm{C}$, whereas the wildtype allele shows a peak of app. $46^{\circ} \mathrm{C}$. One sample is heterozygous for this variant, and the two other samples show the wildtype allele. d Detection of rs786204420 (c.926dupG) via melting analysis (Channel 465-510). Wildtype allele gives a melting peak at app. $65^{\circ} \mathrm{C}$ and the insertion at app. $72^{\circ} \mathrm{C}$. Two samples are wildtype for the tested variant, with a melting point at $65^{\circ}$ $\mathrm{C}$, one sample is homozygous for the c.926dupG giving a melting peak at $72{ }^{\circ} \mathrm{C}$ and one sample is heterozygous for this variant

failure was below $0.01 \%$ (data not shown). A repetition of the qPCR reaction was only necessary after technical failure, e.g., incomplete sealing of the qPCR plate.

\section{PCR results}

From January 2018 to end of July 2019 we screened 291, 905 newborns. For 291, 023 samples no deletion or insertion could be detected in the qPCR screening and were immediately reported as healthy.

Two samples were positive for the homozygous deletion of the $57 \mathrm{~kb}$ region confirmed by an independent laboratory using MLPA analysis (Bioscientia Institut für Medizinische Diagnostik GmbH Zentrum für Humangenetik, Ingelheim, Germany). The diagnosis cystinosis was confirmed by increased free cystine in isolated leukocytes $(2.82 \mathrm{nmol} / \mathrm{mg}$ leukocyte protein [norm $<0.2$ ] for the first case, and in a similar range for the second). 


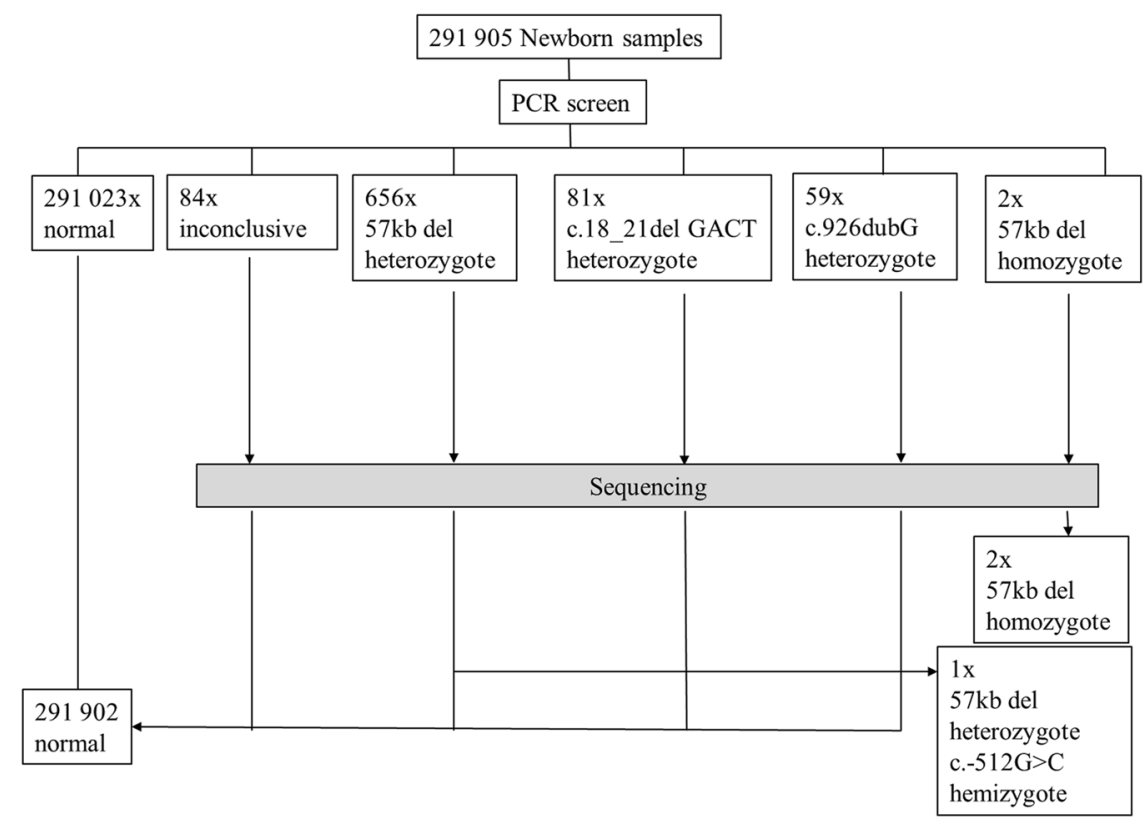

Fig. 2 Results after 19 months of CTNS screening. Within 19 months of screening, 291, 905 samples were analyzed. 291,023 (99.7\%) were negative for any of the three variants in the qPCR assay and reported immediately. $656(0.22 \%)$ samples were positive for a heterozygous $57 \mathrm{~kb}$ deletion, $84(0.028 \%)$ showed inconclusive results and were further analyzed by sequencing. $81(0.027 \%)$ and $59(0.02 \%)$ samples

Six hundred and fifty-six samples were heterozygous for the $57 \mathrm{~kb}$ deletion, 81 for the c.18_21del GACT deletion, and 59 samples for the c.926dupG duplication. Eighty four showed inconclusive results due to shifted melting curves. For all these samples, the test was repeated and the results confirmed (Fig. 2).

\section{Sequencing validation}

All twenty samples from qPCR validation were used for the verification of the sequencing assay. The gene variations identified in the qPCR assay were confirmed-indirectly for the heterozygous $57 \mathrm{~kb}$ deletion, and directly for rs786204501 and rs786204420. For the remaining eight samples missing a second variant, heterozygous gene modifications described as disease causing in the $\mathrm{HGMD}^{\circledR}$ database were found by sequencing (Table 1). For one sample having no disease-causing allele in the qPCR test, a homozygous variant described as disease-causing in the HGMD $^{\circledR}$ database was identified (Table 1). All results were in concordance with the previous analysis.

\section{Sequencing results}

The two homozygous, all heterozygous and samples showing shifted melting peaks were prepared for sequencing, which was done once a week in a batch of were heterozygous for c.18_21del GACT or c.926dupG and sequenced as well. Three potential cystinosis patients could be identified. Two with a homozygous deletion of the $57 \mathrm{~kb}$ region, the other sample compound heterozygous $57 \mathrm{~kb}$ del with a second variant, previously described as disease causing. All others were reported without pathological findings

10-20 samples. Sequencing confirmed the results from the previous qPCR assay. The variations c.18_21del GACT and c.926dupG were found in the respective samples. Even the $57 \mathrm{~kb}$ heterozygous deletion could be identified, as for the deleted region no heterozygous variants were seen giving a hint for an allelic depletion. One sample had a second variant at position c.-512G $>\mathrm{C}$ (rs112140949) in addition to the heterozygous deletion of the $57 \mathrm{~kb}$ region. This genetic constellation was previously described as disease-causing [23].

Beside the previously identified heterozygous modification, two other putative variants described in the $\mathrm{HGMD}^{\circledR}$ as affecting the function of cystinosin could be identified. Both samples showed a shift in the melting analysis of the qPCR assay (Fig. 3b, c). One sample was heterozygous for the c. $922 \mathrm{G}>\mathrm{A}$ variant and the second one for c.29T $>$ C. All remaining samples had no clinically relevant variants (Fig. 3a).

\section{Discussion}

Cystinosis was first described by Abderhalden in 1903 [4]. It is a disease partly covered by the Wilson and Jungner criteria for NBS [24]. In 2008, Nesterova and Gahl pointed out that for optimal cystinosis patient care, a team consisting of nephrologists, metabolic disease, and other specialists 
Table 1 Results of the patient samples used for assay validation

\begin{tabular}{|c|c|c|c|}
\hline Sample & PCR Result 1. Allele & PCR Result 2. Allele & Sequencing \\
\hline 1 & $57 \mathrm{~kb}$ del & $57 \mathrm{~kb}$ del & \\
\hline 2 & c.[18_21del GACT] & c. $\left[18 \_21 \mathrm{del}\right.$ GACT] & \\
\hline 3 & $57 \mathrm{~kb}$ del & $57 \mathrm{~kb}$ del & \\
\hline 4 & c.[926dupG] & c. [926dupG] & \\
\hline 5 & $57 \mathrm{~kb}$ del & c.[18_21del GACT] & \\
\hline 6 & $57 \mathrm{~kb}$ del & $57 \mathrm{~kb}$ del & \\
\hline 7 & $57 \mathrm{~kb}$ del & c.[926dupG] & \\
\hline 8 & $57 \mathrm{~kb}$ del & Neg & c.[696_697dupCG] hemizygous \\
\hline 9 & $57 \mathrm{~kb}$ del & $57 \mathrm{~kb}$ del & \\
\hline 10 & $57 \mathrm{~kb}$ del & Neg & c.790_791delCA hemizygous \\
\hline 11 & $57 \mathrm{~kb}$ del & Neg & c. $1015 \mathrm{G}>\mathrm{A}$ heterozygous \\
\hline 12 & $57 \mathrm{~kb}$ del & $\mathrm{Neg}$ & c. $852 \mathrm{G}>\mathrm{T}$ hemizygous \\
\hline 13 & c. $\left[18 \_21 \mathrm{del}\right.$ GACT] & c.[18_21del GACT] & \\
\hline 14 & $57 \mathrm{~kb}$ del & Neg & c. $923 \mathrm{G}>\mathrm{T}$ hemizygous \\
\hline 15 & $57 \mathrm{~kb}$ del & Neg & c.790_791delCA hemizygous \\
\hline 16 & $57 \mathrm{~kb}$ del & Neg & c.295_310del16 hemizygous \\
\hline 17 & c.[18_21del GACT] & c.[18_21del GACT] & \\
\hline 18 & $57 \mathrm{~kb}$ del & Neg & c.[696_697dupCG] hemizygous \\
\hline 19 & neg & Neg & c.516dupC homozygous \\
\hline 20 & c.[18_21del GACT] & c.[18_21del GACT] & \\
\hline
\end{tabular}

$57 \mathrm{~kb}$ del refers to NC_000017.11:g.3600934_3658165del (GRCh38) is necessary. In addition patients should be treated in specialized centers to improve their care and quality of life [24]. These, as well as other authors also highlighted that early diagnosis is particularly important for an effective therapy. They therefore propose to develop a test for NBS [4].

Biochemical diagnosis is usually done by determining the concentration of free cystine in isolated leukocytes, which is not possible from DBS. Genetic analysis of DNA extracted from DBS is hampered by the relatively high number of variants responsible for the disease. Analysis could be performed by whole genome/exome (WGS/ WES) or amplicon based sequencing for a limited number of genes. However, the first two methods are ethically questionable, all are very expensive and have a long turnaround time compared with tandem mass spectrometry [3, 18]. In addition they are not capable to handle 600-1,200 samples per day, leading to first results within $24 \mathrm{~h}$. In the near future, turnaround time and costs will be manageable with the development of alternative protocols and the introduction of novel sequencing technologies. Nevertheless, ethical and reporting issues remain. These include the handling of incidental findings, storage time of sequencing information, and the question of reanalysis of older data after discovery of novel clinical variants $[1,14]$. The establishment of appropriate guidelines might help to resolve these issues.
A recent publication describes the variant pattern of the CTNS gene in Germany [11]. In combination with data of 80 cystinosis patients identified in Germany (Harms et al., unpublished), the allele frequencies of these variants were calculated. The most common one is the homozygous deletion of a $57 \mathrm{~kb}$ region, including most of the CTNS gene with an allelic frequency of 57.5-65.7\%. The second most frequent variant leads to a frameshift (c.18_21del GACT) and has a frequency of 8.6-15\%. The third most prominent gene variation is a duplication of a single guanosine at position c.926, showing a frequency of $8-10 \%$. Our results from the screening, regarding the proportions of the three variations, fit the expected range, i.e., $57 \mathrm{~kb}$ del $(N=656)$, c.18_21del GACT $(N=81)$, and c.926dupG $(N=59)$.

A screening approach beginning with the identification of the carriers of these three major variants in a first step will also cover all compound heterozygotes by the subsequent investigation using NGS and thus achieve a total sensitivity of $>90 \%$ of all cystinosis patients.

Based on the Hardy-Weinberg calculation, 69-74\% of the patients are homozygous or compound heterozygous for one or two of these three variants. However, $26-31 \%$ of the patients would have only one of these three gene modifications and a secondary different one. Therefore we decided to sequence CTNS from all heterozygous samples as second-tier approach to increase test sensitivity. Only 

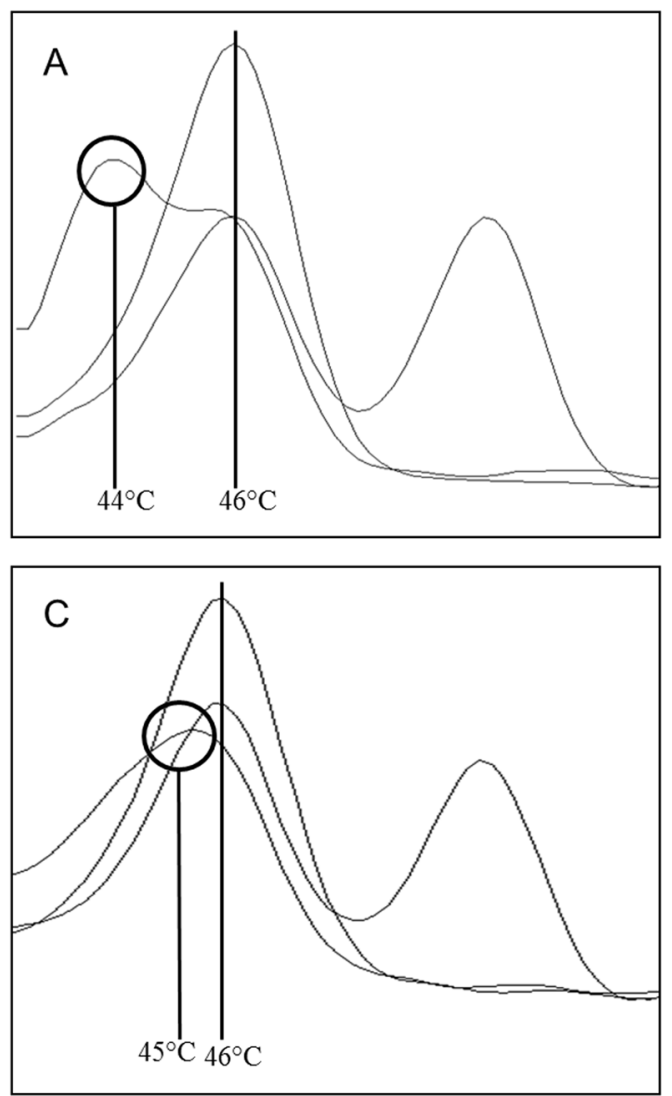

Fig. 3 Shift of melting peaks. a Melting analysis to detect rs786204501 (c.18_21del GACT) in the channel 498-640 with a heterozygous sample showing a lowered melting peak $\left(44^{\circ} \mathrm{C}\right)$ next to the wildtype peak $\left(46^{\circ} \mathrm{C}\right)$. Sequencing identified the $\mathrm{c} .12 \mathrm{~T}>\mathrm{C}$ variant, described as non-pathogenic. The two other samples showed a wildtype and a heterozygous melting peak. b Melting analysis to detect rs786204420 (c.926dupG) in the channel 465-510 with a heterozygous sample showing a lowered melting peak $\left(62^{\circ} \mathrm{C}\right)$ next to the wildtype $\left(65^{\circ} \mathrm{C}\right)$. Sequencing identified the c.922G $>A$ variant, described as disease-causing in $\mathrm{HGMD}^{\circledR}$ database. One sample is

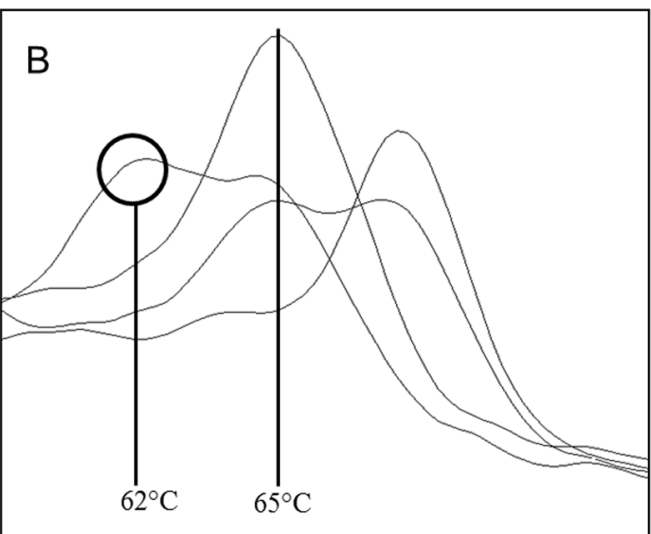

wildtype for the tested variant, with a melting point at $65^{\circ} \mathrm{C}$, one sample is homozygous for the c.926dupG giving a melting peak at $72{ }^{\circ} \mathrm{C}$ and one sample is heterozygous for this variant. c Melting analysis to detect rs786204501 (c.18_21del GACT) in the channel 498-640 with a heterozygous sample showing a lower and more wide melting peak $\left(45^{\circ} \mathrm{C}\right)$ relative to the wildtype peak $\left(46^{\circ} \mathrm{C}\right)$. Sequencing identified the c.29T $>\mathrm{C}$ variant, described as disease-causing in $\mathrm{HGMD}^{\circledR}$ database. The two other samples showed a wildtype and a heterozygous melting peak

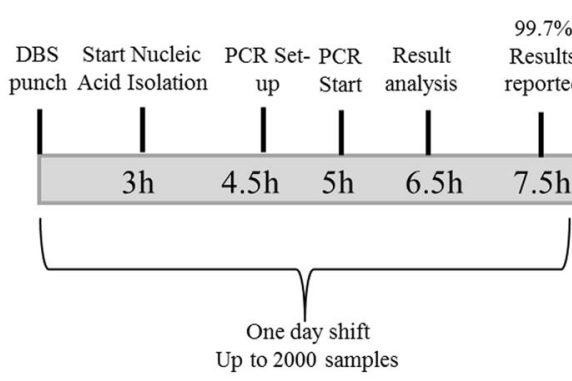

Fig. 4 Time frame of cystinosis screening. Up to 2,000 samples can be processed with this approach, allowing reporting results for $99.7 \%$ of all samples in one day. Homo- or compound heterozygous status for

previously described variants marked to be pathologic in the HGMD $^{\circ}$ were included in the sequencing analysis (120 variations as by January 2018). Until January 2019 more than 140 variants were described [25].

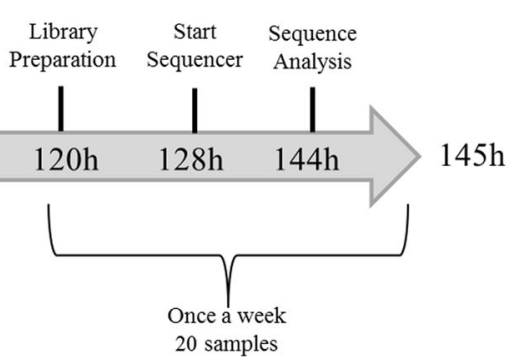

the three variations screened for, are confirmed by retesting. Secondtier diagnostic by next-generation sequencing (NGS) provides results within six days for the oldest samples

The goal of our DNA-based screening attempt was to report first-tier results within the same time frame as for conventional screening methods like MS/MS, thus delivering same-day results. With a newly developed protocol 
for nucleic acid isolation [19] followed by qPCR in 384well format, we were able to provide first-tier results within $7.5 \mathrm{~h}$ for up to 2,000 samples. Only $0.25 \%$ of these needed to be sequenced in a second-tier analysis providing final results within $145 \mathrm{~h}$ (Fig. 4).

Using NGS as a second-tier test does not increase overall screening costs, since only small numbers of all samples need to be sequenced. In contrast to WGS/WES approaches, amplicon-based NGS of one gene, like CTNS, further reduces costs and the results can be interpreted without the need of complicated bioinformatics. To our knowledge, there is no library preparation protocol for WGS/WES available so far, leading to sequencing results within a feasible timeframe for NBS.

Nevertheless, there might be alternative methods to NGS, like Sanger sequencing or chip hybridization [26]. However, the amount of time and reagents required to analyze 29 amplicons in two directions of 10-20 samples is significantly higher using Sanger sequencing. Chip hybridization on the other hand is not as flexible, since it cannot be easily extended to other polymorphisms.

Our primary PCR screening depends on the presence of one of the three main variants found in the German population. The $57 \mathrm{~kb}$ deletion is the most prominent one, found in $\sim 75 \%$ of the known patients in Germany. Similar to other genetic disorders with a founder effect in Northern Europe, it shows a clear north-south gradient. The deletion is only rarely found in southern Italy [27] and not at all in Turkey, Africa, Asia, or the Middle East [28]. Therefore the primary screening approach by qPCR has to be adapted by changing PCR targets, regarding the geographical region. Keeping the high Turkish population in Germany in mind as well as the immigration during recent years, the sensitivity of our PCR screen might be limited. But, so far no further cases of cystinosis were reported to us, nevertheless earliest symptoms were usually diagnosed by the age of 8-12 month, and therefore it might be too early to determine the sensitivity of the screening program.

We identified two newborns with a homozygous $57 \mathrm{~kb}$ deletion and a second one with heterozygous $57 \mathrm{~kb}$ deletion and a $\mathrm{G}>\mathrm{C}$ substitution at position c.-512 on the second allele, putatively leading to promoter dysfunction [23]. This is in concordance with the expected prevalence of $1: 100,000$ to $1: 200,000$. For the two cases having a homozygous deletion cystinosis was confirmed by determination of free cystine concentration in leukocytes. For the third newborn no increase of free cystine in leukocytes was detectable so far. Phornphutkul et al. described a patient with the same genetic constellation, showing increased cystine concentrations as well as other distinct clinical symptoms present in cystinosis patients. A significant difference in both cases it the age of diagnosis.
The latter patient [23] was already several years old, and it could be possible that the promoter variant c. $-512 \mathrm{G}>\mathrm{C}$ leads to a delayed start of symptoms. Nevertheless, it cannot be ruled out, that this modification does not affect the function at all, and the authors missed a second harmful gene modification. The newborn will be closely monitored in the future to detect symptoms as early as possible.

For the first time we have shown that a combination of qPCR and NGS can be used in a routine setting for NBS. Thus, we suggest that a fast, cost effective screening is manageable for diseases caused by a limited number of high and a higher number of low prevalence variants in such a two-tiered approach.

Acknowledgements We highly appreciate the help of Prof. Dr Carsten Bergmann and Dr Neuber in selecting clinically relevant variants in the CTNS gene.

Funding The screening was funded by the Cystinose Foundation (Cystinose Stifung, DSZ-Regional Office Munich; Widenmayerstr. 10; 80538 Munich; Germany) and the Dietmar Hopp Foundation (Raiffeisenstraße 51, 68789 St. Leon-Rot, Germany). The funders played no role in design, interpretation, and publication of this study.

\section{Compliance with ethical standards}

Conflict of interest The authors declare that they have no conflict of interest.

Publisher's note Springer Nature remains neutral with regard to jurisdictional claims in published maps and institutional affiliations.

\section{References}

1. Ficicioglu C. New tools and approaches to newborn screening: ready to open Pandora's box? Cold Spring Harb Mol Case Stud. 2017;3:a001842.

2. Guthrie R, Susi A. A simple phenylalanine method for detecting phenylketonuria in large populations of newborn infants. Pediatrics. 1963;32:338-43.

3. Berg JS, Agrawal PB, Bailey DB Jr., Beggs AH, Brenner SE, Brower AM, et al. Newborn sequencing in genomic medicine and public health. Pediatrics. 2017;139:e20162252.

4. Elmonem MA, Veys KR, Soliman NA, van Dyck M, van den Heuvel LP, Levtchenko E. Cystinosis: a review. Orphanet J Rare Dis. 2016;11:47.

5. Nesterova G, Gahl WA. Cystinosis: the evolution of a treatable disease. Pediatr Nephrol. 2013;28:51-9.

6. Baumner S, Weber LT. Nephropathic cystinosis: symptoms, treatment, and perspectives of a systemic disease. Front Pediatr. 2018;6:58.

7. Bendavid C, Kleta R, Long R, Ouspenskaia M, Muenke M, Haddad $\mathrm{BR}$, Gahl WA. FISH diagnosis of the common 57-kb deletion in CTNS causing cystinosis. Hum Genet. 2004;115:510-4.

8. Buntinx L, Voets T, Morlion B, Vangeel L, Janssen M, Cornelissen E, et al. TRPV1 dysfunction in cystinosis patients harboring the homozygous $57 \mathrm{~kb}$ deletion. Sci Rep. 2016;6:35395.

9. Forestier L, Jean G, Attard M, Cherqui S, Lewis C, van't Hoff W, et al. Molecular characterization of CTNS deletions in 
nephropathic cystinosis: development of a PCR-based detection assay. Am J Hum Genet. 1999;65:353-9.

10. Kalatzis V, Cherqui S, Jean G, Cordier B, Cochat P, Broyer M, Antignac C. Characterization of a putative founder mutation that accounts for the high incidence of cystinosis in Brittany. J Am Soc Nephrol. 2001;12:2170-4.

11. Kiehntopf M, Schickel J, Gonne B, Koch HG, Superti-Furga A, Steinmann B, et al. Analysis of the CTNS gene in patients of German and Swiss origin with nephropathic cystinosis. Hum Mutat. 2002;20:237.

12. Topaloglu R, Vilboux T, Coskun T, Ozaltin F, Tinloy B, GunayAygun M, et al. Genetic basis of cystinosis in Turkish patients: a single-center experience. Pediatr Nephrol. 2012;27:115-21.

13. Sommerburg O, Stahl M, Hammermann J, Okun JG, Kulozik A, Hoffmann G, Mall M. Newborn screening on cystic fibrosis in germany: comparison of the new screening protocol with an alternative protocol. Klin Padiatr. 2017;229:59-66.

14. Kwon JM, Matern D, Kurtzberg J, Wrabetz L, Gelb MH, Wenger $\mathrm{DA}$, et al. Consensus guidelines for newborn screening, diagnosis and treatment of infantile Krabbe disease. Orphanet J Rare Dis. 2018;13:30.

15. Chien YH, Chiang SC, Weng WC, Lee NC, Lin CJ, Hsieh WS, et al. Presymptomatic diagnosis of spinal muscular atrophy through newborn screening. J Pediatr. 2017;190:124-9.

16. Hao Z, Fu D, Ming Y, Yang J, Huang Q, Lin W, et al. Large scale newborn deafness genetic screening of 142,417 neonates in Wuhan, China. PLoS ONE. 2018;13:e0195740.

17. Nakagawa H, Fujita M. Whole genome sequencing analysis for cancer genomics and precision medicine. Cancer Sci. 2018;109:513-22.

18. Kingsmore SF. Newborn testing and screening by whole-genome sequencing. Genet Med. 2016;18:214-6.

19. Czibere L, Burggraf S, Fleige T, Glück B, Keitel LM, Landt O et al. High-throughput genetic newborn screening for spinal muscular atrophy by rapid nucleic acid extraction from dried blood spots and 384-well qPCR. Eur J Hum Genet 2019. https://doi.org/10.1038/s41431-019-0476-4.

20. Anikster Y, Lucero C, Touchman JW, Huizing M, McDowell G, Shotelersuk V, et al. Identification and detection of the common 65-kb deletion breakpoint in the nephropathic cystinosis gene (CTNS). Mol Genet Metab. 1999;66:111-6.

21. Robinson JT, Thorvaldsdottir H, Winckler W, Guttman M, Lander ES, Getz G, Mesirov JP. Integrative genomics viewer. Nat Biotechnol. 2011;29:24-6.

22. Thorvaldsdottir H, Robinson JT, Mesirov JP. Integrative Genomics Viewer (IGV): high-performance genomics data visualization and exploration. Brief Bioinform. 2013;14:178-92.

23. Phornphutkul C, Anikster Y, Huizing M, Braun P, Brodie C, Chou JY, Gahl WA. The promoter of a lysosomal membrane transporter gene, CTNS, binds Sp-1, shares sequences with the promoter of an adjacent gene, CARKL, and causes cystinosis if mutated in a critical region. Am J Hum Genet. 2001;69:712-21.

24. Nesterova G, Gahl W. Nephropathic cystinosis: late complications of a multisystemic disease. Pediatr Nephrol. 2008;23:863-78.

25. David D, Princiero Berlingerio S, Elmonem MA, Oliveira Arcolino F, Soliman N, van den Heuvel B, et al. Molecular basis of cystinosis: geographic distribution, functional consequences of mutations in the ctns gene, and potential for repair. Nephron. 2019;141:133-46.

26. Dogan M, Bulan K, Kaba S, Cesur Y, Ceylaner S, Ustyol L. Cystinosis in Eastern Turkey. J Pediatr Endocrinol Metab. 2016;29:965-9.

27. Mason S, Pepe G, Dall'Amico R, Tartaglia S, Casciani S, Greco $\mathrm{M}$, et al. Mutational spectrum of the CTNS gene in Italy. Eur J Hum Genet. 2003;11:503-8.

28. Sadeghipour F, Basiratnia M, Derakhshan A, Fardaei M. Mutation analysis of the CTNS gene in Iranian patients with infantile nephropathic cystinosis: identification of two novel mutations. Hum Genome Var. 2017;4:17038. 